\title{
Interferência da Indústria do Tabaco no Brasil: a Necessidade do Ajuste de Contas
}

\author{
doi: https://doi.org/10.32635/2176-9745.RBC.2020v66n2.878
}

\author{
Tobacco Industry Interference in Brazil: the Necessity of Settling Accounts \\ Interferencia de la Industria Tabacalera en Brasil: la Necesidad del Ajuste de Cuentas
}

André Salem Szklo'; Felipe Lacerda Mendes²; Tânia Maria Cavalcante³; João Ricardo Viegas ${ }^{4}$

Resumo

Introdução: No Brasil, cada vez mais são identificadas açóes ilegais de publicidade, propaganda e patrocínio por parte da indústria do tabaco em eventos musicais e por meio das redes sociais, voltadas a atrair principalmente o público jovem para o uso do cigarro. Objetivo: Desenvolver uma metodologia que permita estabelecer um parâmetro de quantificação dos impactos negativos para o setor saúde desse descumprimento da lei. Método: Combinaram-se as informaçôes nacionais existentes sobre i) a equivalência entre "custo direto médio da assistência médica" e "mortes por doenças atribuíveis ao tabagismo" e ii) a equivalência entre "a parcela do lucro revertido em açóes de marketing" e "mortes de fumantes que contribuíram para a geração desse lucro por meio da compra de cigarros", de forma a se obter a relação "custo direto do tratamento" vs "parcela do lucro revertido em açóes de marketing". As doenças selecionadas foram aquelas que apresentam os maiores custos diretos de tratamento atribuíveis ao fumo. Resultados: Para cada centavo investido em marketing pela indústria do tabaco, o Brasil tem um gasto com tratamento de doenças relacionadas ao tabaco 1,93 vezes superior ao dinheiro investido pela indústria. Conclusáo: A mensuração da responsabilização dos violadores da legislação nacional para o controle do tabaco é fundamental para compensar parte dos custos associados ao tratamento de pacientes e aos programas de cessação ao fumo, favorecendo assim a redução do tabagismo no país.

Palavras-chave: Indústria do Tabaco; Publicidade de Produtos Derivados do Tabaco; Tabagismo/economia; Compensação e Reparação; Marketing Social.

\begin{abstract}
Introduction: In Brazil, illegal actions of advertising, promotion, and sponsorship by part of the tobacco industry are increasingly identified in music events, and through social media, aimed mainly to attract young people to use cigarettes. Objective: To develop a methodology that allows the creation of a parameter of quantification of the negative impacts to the health sector of non-compliance with the law. Method: Combination of the current national information about i) the equivalence between "mean direct cost of medical care" and "deaths by diseases attributable to tobacco addiction" and ii) the equivalence between "the portion of the profit translated into marketing actions" and "deaths of smokers who contributed for the generation of this profit through purchase of cigarettes" in order to obtain the relation between "direct cost of the treatment" vs "portion of the profit translated into market actions". The diseases selected were those that presented the biggest direct cost of treatment attributable to tobacco. Results: For every cent invested in marketing strategies by the tobacco industry, Brazil spends 1.93 times more financial resources to treat tobacco-related diseases. Conclusion: The measurement of the liability for non-compliance of the tobacco national legislation is essential to offset part of the associated costs of the treatment of patients and programs of tobacco cessation to favor the reduction of smoking prevalence in Brazil.

Key words: Tobacco Industry; Tobacco-Derived Products Publicity; Tobacco Use Disorder/economics; Compensation and Redress; Social Marketing.
\end{abstract}

Resumen

Introducción: En Brasil, es cada vez más común identificar acciones ilegales de publicidad, promoción y patrocinio del tabaco por parte de la industria tabacalera en eventos musicales y a través de redes sociales, destinadas principalmente a atraer al público joven al consumo de cigarrillos. Objetivo: Desarrollar una metodología que permita establecer un parámetro para cuantificar los impactos negativos al setor de la salud de esa acción ilegal de la ley. Método: El artículo integra la información nacional existente sobre i) la equivalencia entre el "costo directo promedio de asistencia médica" y "muertes por enfermedades atribuibles al tabaquismo" y ii) la equivalencia entre "la parte del ingreso usado en acciones de marketing" y "las muertes de fumadores que han contribuido a la generación de estos ingresos a través de la compra de cigarrillos", para obtener la relación "costo directo del tratamiento" vs "parte de los ingresos usados en acciones de marketing". Las enfermedades seleccionadas fueron las que presentaron los costos más altos de tratamiento directo atribuibles al uso del tabaco. Resultados: Por cada centavo invertido en marketing por la industria tabacalera, Brasil tiene un gasto en tratamiento de enfermedades relacionadas con el tabaco 1,93 veces mayor que el monto invertido por la industria. Conclusión: Medir la responsabilidad de los infractores de la legislación nacional de control del tabaco es esencial para compensar parte de los costos asociados con el tratamiento de los pacientes y com los programas para dejar de fumar, favoreciendo así la reducción del consumo de tabaco en el país.

Palavras clave: Industria del Tabaco; Publicidad de Productos Derivados del Tabaco; Tabaquismo/economia; Compensación y Reparación; Mercadeo Social.

1 Divisão de Pesquisa Populacional. Instituto Nacional de Câncer José de Alencar Gomes da Silva (INCA). Rio de Janeiro (RJ), Brasil. Orcid iD: https://orcid.org/00000003-1903-6188

${ }^{2}$ Comissão Nacional para Implementação da Convenção-Quadro no Brasil. INCA. Rio de Janeiro (RJ), Brasil. Orcid iD: https://orcid.org/0000-0003-1071-1597 ${ }^{3}$ Comissão Nacional para Implementação da Convenção-Quadro no Brasil. INCA. Rio de Janeiro (RJ), Brasil. Orcid iD: http://orcid.org/0000-0001-8556-9949

${ }^{4}$ Coordenador Regional das Américas para a Convenção-Quadro para o Controle do Tabaco e para o Protocolo para Eliminar o Comércio llícito de Produtos de Tabaco. Assessoria Internacional. INCA. Rio de Janeiro (RJ), Brasil. Orcid iD: https://orcid.org/0000-0002-0571-2969

Endereço para correspondência: André Salem Szklo. Rua Marquês de Pombal, 125 - Centro. Rio de Janeiro (RJ), Brasil. CEP 20230-240. E-mail: aszklo@inca.gov.br 


\section{INTRODUÇÃO}

Atualmente, há 1,1 bilhão de fumantes no mundo sob maior risco de desenvolverem doenças graves causadas pelo tabagismo do que os náo fumantes, tais como câncer, doenças cardiovasculares e respiratórias crônicas ${ }^{1,2}$. Consequentemente, o tabagismo é responsável por mais de oito milhóes de mortes anuais no mundo, sendo sete milhóes o resultado direto do consumo do tabaco, enquanto cerca de 1,2 milhão é decorrente de não fumantes expostos ao fumo passivo ${ }^{2}$. No Brasil, o tabagismo causa 157 mil mortes ao ano, o que corresponde a $12,6 \%$ do total das mortes anuais ${ }^{3}$, sendo responsável por $21,4 \%$ de todas as mortes por câncer ${ }^{4}, 74 \%$ das mortes por doença pulmonar obstrutiva crônica (DPOC), 18\% das mortes por doenças coronarianas (angina e infarto) e $13 \%$ das mortes por doenças cerebrovasculares (acidente vascular cerebral $-\mathrm{AVC})^{3}$. Vale a pena assinalar, ainda, que o tabagismo por si só é também considerado uma doença e, como tal, está inserido na décima edição da Classificação Internacional de Doenças e Problemas Relacionados à Saúde (CID-10) da Organização Mundial da Saúde (OMS) 5 .

Para enfrentar essa epidemia global, houve o estabelecimento do primeiro tratado internacional de saúde pública com efeitos vinculantes da história da OMS, a Convençáo-Quadro da OMS para o Controle do Tabaco (CQCT/OMS) $)^{6}$ Seu objetivo é "proteger as geraçóes presentes e futuras das devastadoras consequências sanitárias, sociais, ambientais e econômicas geradas pelo consumo e pela exposição à fumaça do tabaco". Uma das açôes efetivas para reduzir a demanda por tabaco está prevista no compromisso disposto no Artigo 13 da CQCT/OMS 6 . Neste, as Partes se comprometem a proceder com a proibição total de toda forma publicidade, promoção e patrocínio do tabaco.

A relevância desse compromisso para a redução do número de usuários de tabaco consta simbolizada nas palavras da então Diretora-Geral da OMS, Gro Brundtland, ao destacar que "o tabagismo seria uma doença transmissível; transmissível a partir da mídia, da indústria do entretenimento e mais diretamente a partir das açôes de marketing e promoção de produtos de tabaco pela indústria". Assim sendo, formas explícitas de sua associação com glamour, prazer, desempenhos físico e sexual, entre outras, acabam por incentivar a iniciaçáo de jovens e adolescentes ao consumo de produtos que liberam substâncias psicoativas e geram uma dependência química e transtorno mental ${ }^{8,9}$.

De fato, em documentos internos confiscados por litígios nos Estados Unidos da América e no Reino Unido, fica muito claro como as empresas transnacionais, que operam também no Brasil, aprimoraram a sua capacidade de induzir adolescentes ao tabagismo, sendo essa iniciação ao fumo fundamental para a "rentabilidade do negócio" (e.g., "O adolescente de hoje é o potencial cliente regular de amanhã e a esmagadora maioria dos fumantes começa a fumar ainda na adolescência. Os padróes de tabagismo dos adolescentes são particularmente importantes para a Philip Morris"; tradução nossa) $)^{9}$.

Desde 2005, o Brasil é Estado Parte da CQCT/ OMS, o que deu impulso às medidas de controle do tabagismo que já vinham sendo implementadas no país desde a década de 1990. Nesse cenário, merece destaque o Artigo 220 da Constituição Federal de 1988 que abriu a possibilidade de restriçóes da propaganda, comercial de produtos de tabaco, por meio da Lei Federal n. ${ }^{\circ}$ 9.294 de $1996^{10}$. Essa Lei, que inicialmente restringia os horários de veiculação das propagandas nos principais meios de comunicação, foi paulatinamente modificada por outras leis federais que ampliaram essas restriçôes, até atingir a proibição da propaganda de produtos de tabaco em $2011^{10}$. Dessa forma, atualmente no país, estão proibidas a propaganda pró-tabaco em televisão, rádio, jornais, revistas, Internet, eventos de arte e esportivos, restando somente autorizada a exposição dos produtos derivados do tabaco nos pontos de venda, sob uma série de restriçóes específicas definidas pela Agência Nacional de Vigilância Sanitária (Anvisa) ${ }^{11}$. Cabe destaque a obrigação de inclusão de advertência sanitária sobre os malefícios do tabagismo à saúde nos mostruários e expositores de produtos de tabaco estabelecidos nos pontos de venda.

Essas açôes, somadas às demais medidas da CQCT/ OMS implementadas no Brasil, tais como taxação de produtos derivados do tabaco, implementação de ambientes coletivos livres de fumo, promoção da cessação de fumar, e implementação de campanhas de esclarecimento à população sobre os males do tabagismo, geraram importantes resultados em termos de redução da prevalência de fumantes, inclusive entre jovens, e, consequentemente, também de diminuição das mortes causadas pelo tabagismo (e.g., redução de cerca de 60\% na proporção de fumantes adultos em 15 anos: de 34,6\% em 1989 para $14,7 \%$ em 2013) $)^{12,13}$.

Apesar de todo o esforço, no Brasil, cerca de 19\% dos adolescentes entre 13 e 15 anos ainda experimentam cigarros $^{14}$ instigados por estratégias de marketing mantidas pelos fabricantes de cigarros. Os adolescentes que estáo experimentando os cigarros são aqueles mencionados nos documentos internos da indústria, sendo que uma parte deles continuará a fumar na vida adulta ${ }^{12,14}$. Tal situação contribui, assim, para "um movimento cíclico perverso" no qual a perpetuação do lucro da indústria do tabaco se dá 
às custas do adoecimento e morte dos seus consumidores atuais, vítimas de uma dependência química iniciada na infância e na adolescência e, consequentemente, do efeito cumulativo do tabagismo ao longo do tempo ${ }^{1}$.

A OMS estima que o consumo do cigarro mate dois em cada três fumantes ${ }^{15}$, incorrendo, consequentemente, também em gastos de tratamento de saúde e de perda de produtividade $^{3}$. Estima-se ainda que o custo do tabaco para a economia global seja próximo a US $\$ 2$ trilhóes por ano, i.e., aproximadamente $2 \%$ do Produto Interno Bruto (PIB) global ${ }^{16,17}$. Já no Brasil, um estudo sobre a carga de doença atribuível ao uso do tabaco apontou que os custos diretos da assistência médica relacionados ao tabagismo equivalem a cerca de $8,0 \%$ de todo gasto público e privado em saúde do país, sendo que, quando são adicionados os custos indiretos em virtude da produtividade perdida por morte prematura e incapacidade, isso equivale a $0,96 \%$ de todo o PIB nacional ${ }^{3,18}$. E a arrecadação de impostos do setor tabaco cobre apenas cerca de $23 \%$ das perdas geradas pelo tabagismo ${ }^{3}$.

Este artigo tem, portanto, como objetivo desenvolver uma metodologia que permita, a partir das pesquisas nacionais existentes sobre o comportamento do fumante, estabelecer uma relação entre a parcela do lucro da indústria do tabaco revertido em açôes de marketing para atrair novos fumantes e os gastos decorrentes com o tratamento das doenças relacionadas ao tabaco dos fumantes atuais.

\section{MÉTODO}

O presente artigo propóe uma metodologia que permita combinar as informaçôes de dois estudos apresentados detalhadamente em trabalhos já publicados ${ }^{3,15}$.

Estudo I: Equivalência entre lucro revertido em açóes de marketing e mortes de fumantes que contribuíram para a geração desse lucro ${ }^{15}$.

Vender cigarros e outros produtos de tabaco é o propósito das empresas do ramo fumageiro e, como qualquer empresa, seu objetivo maior é aumentar seus lucros e ampliar sua fatia de mercado. Para tanto, essas empresas continuam usando cada vez mais uma parcela dos lucros com as vendas dos cigarros em estratégias para minar as políticas de combate ao tabagismo e para captar novos consumidores, garantindo, assim, a expansão de seus negócios ${ }^{19}$. Entre os diversos exemplos de estratégias que buscam distanciar a imagem negativa do seu negócio principal, podem ser citados investimentos em projetos sociais, culturais, ambientais, em eventos e pesquisa ${ }^{19}$.

E quanto custa, portanto, para a sociedade brasileira em termos de mortes esse investimento de marketing da indústria do tabaco? Para responder a essa pergunta, o estudo quantificou o impacto das vendas de cigarros legais em mortes, a partir do seguinte fluxo lógico (Figura 1).

O estudo se baseou em dados sobre o comportamento do fumante brasileiro com idade igual ou superior a 35 anos, no ano de 2013, provenientes da Pesquisa Nacional de Saúde $(\mathrm{PNS})^{20}$ e da Secretaria da Receita Federal ${ }^{21}$,

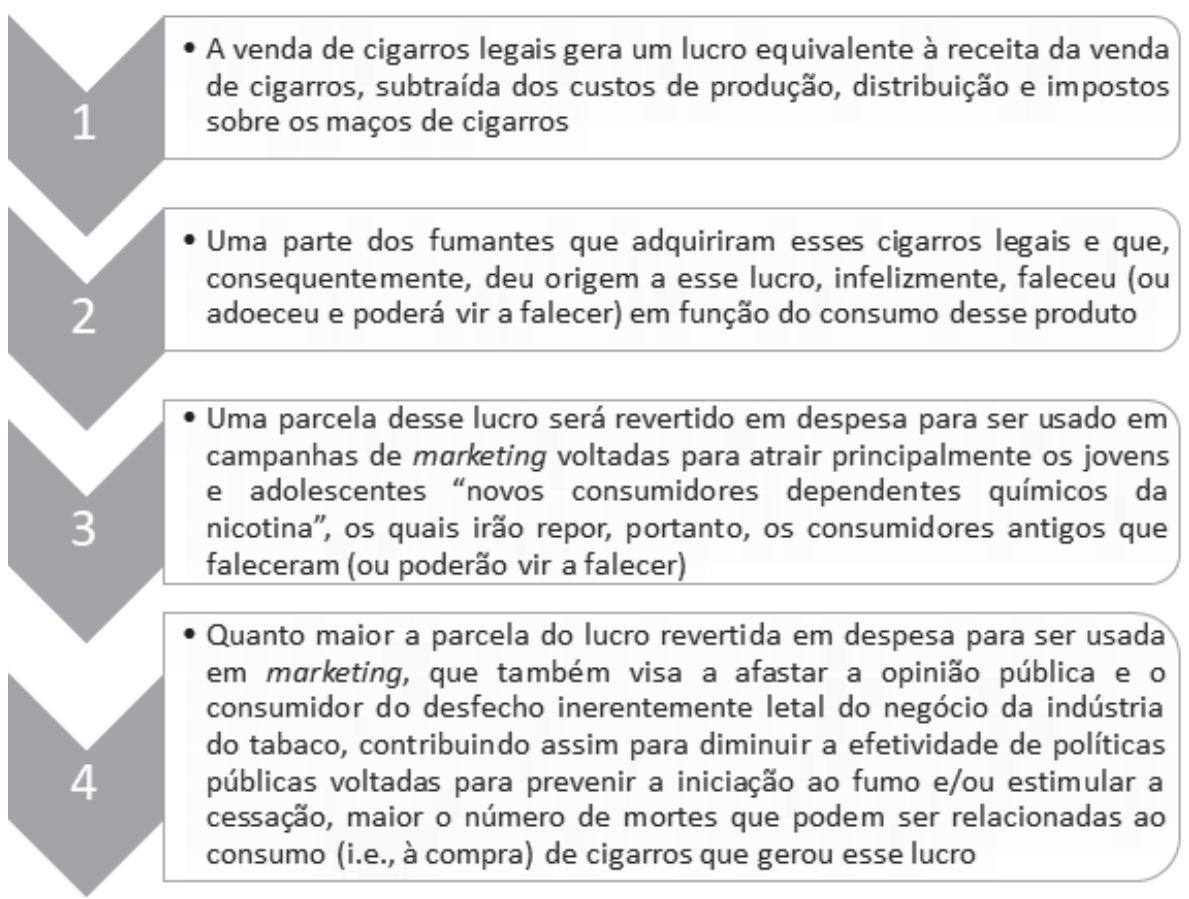

Figura 1. Fluxo lógico da quantificação em mortes de fumantes a partir do lucro oriundo das vendas legais de cigarros e revertido em ações de marketing 
assim como de informaçóes sobre a taxa de mortalidade ajustada pela distribuição etária da população brasileira para doenças específicas, segundo sexo e estado de fumante (fumante, ex-fumantes e não fumantes) ${ }^{22,23}$. Utilizaram-se, ainda, os dados disponíveis de mortalidade específica por doença e sexo da população brasileira ${ }^{23}$.

Mais detalhes sobre a metodologia do estudo envolvendo a estimativa do lucro da indústria do tabaco oriunda da venda de cigarros legais e os cálculos das fraçôes da mortalidade por doenças específicas atribuíveis ao consumo de cigarros legais, dados estes necessários para estabelecer a equivalência entre o lucro revertido integralmente em açôes de marketing e mortes de fumantes que contribuíram para a geração desse lucro total, podem ser encontrados em publicação recente ${ }^{15}$.

Estudo II: Equivalência entre custos diretos da assistência médica das doenças relacionadas ao tabaco e mortes atribuíveis ao tabagismo ${ }^{3}$.

Calculou-se a carga do tabagismo para o país em termos de mortalidade, morbidade e custos diretos da assistência médica de 16 doenças relacionadas ao tabaco em 2015. Para tal, foi desenvolvido um modelo matemático baseado em uma microssimulação probabilística de milhares de indivíduos com 35 anos ou mais de idade ${ }^{24}$, no qual os parâmetros usados pelo modelo foram apoiados: i) na mortalidade geral da população e na mortalidade específica por doença, idade e $\operatorname{sexo}^{23}$; ii) na prevalência de fumantes/ ex-fumantes/não fumantes ${ }^{20}$; iii) nos riscos relativos de desenvolver a doença específica, segundo idade, sexo, e condição de uso do tabaco ${ }^{25}$; e iv) nas letalidades por idade e sexo para as respectivas doenças ${ }^{23,26,27}$. Além disso, para o custo direto da assistência médica, foi estimado o custo médio unitário por doença para o Sistema Único de Saúde (SUS) e para o setor de saúde suplementar por meio da técnica do microcusteio e do custo por procedimento ${ }^{28-32}$.

Mais detalhes sobre a metodologia do modelo e os cálculos dos respectivos custos das doenças podem ser encontrados em publicação recente ${ }^{3}$.

Combinando Estudo I e Estudo II:

As doenças selecionadas para o presente artigo foram aquelas que apresentaram os maiores custos diretos de tratamento atribuíveis ao tabagismo ${ }^{3}$ e que estiveram também presentes nos cálculos do estudo I: doenças cardíacas isquêmicas (CID-10 I20-I25), DPOC (CID-10 J40-J44), AVC (CID-10 I60-I69) e câncer de pulmão (CID-10 C33-C34)

Considerando que os dados do estudo I trazem a possibilidade de reflexão sobre qual o custo em vidas que representa os investimentos em marketing da indústria do tabaco, inclusive por meio de doaçóes como estratégia para formar alianças contra as medidas de reduçáo do tabagismo ${ }^{19}$, pode-se pensar em ir além e passar de um custo "social-moral" para um custo financeiro. Para tal, é necessário dividir a informação de custo direto de tratamento/assistência médica associado às mortes pelo tabagismo, que foi estimado no estudo II, pela equivalência entre o lucro revertido integralmente em despesa com açóes de marketing e as mortes de fumantes que contribuíram para a geração desse lucro total, que foi estimada pelo estudo I. Dessa forma, se estabelece uma relação constante entre "custo direto médio do tratamento" e "despesa potencial para ser usada em açôes de marketing", a qual poderá ser útil nos casos em que se consiga levantar o valor efetivamente gasto com, por exemplo, uma campanha de marketing, a fim de determinar o custo equivalente para o Brasil em termos de tratamento de fumantes.

Vale a pena assinalar que os custos diretos de tratamento/assistência calculados originalmente em Reais (R\$) de 2015 foram corrigidos para 2013 pelo ajuste inflacionário medido pela variação do Índice Nacional de Preços ao Consumidor Amplo (IPCA) ${ }^{33}$ de forma a se utilizar o mesmo ano de referência para, combinar as informações dos estudos I e II.

\section{RESULTADOS}

\section{Estudo I:}

No Brasil, o estudo estimou que, em 2013, cada $\mathrm{R}$ \$ 0,137 milhão de lucro auferido por empresa de tabaco com a venda de cigarros legais, e hipoteticamente usado integralmente para estratégias de marketing, foi equivalente a uma morte por DPOC, câncer de pulmão, infarto agudo do miocárdio, síndrome coronariana aguda ou AVC atribuível ao tabagismo (Tabela 1).

\section{Estudo II:}

Durante o ano de 2015, o tabagismo foi responsável por 93.889 mortes por cinco causas: DPOC, câncer de pulmáo, infarto agudo do miocárdio, síndrome coronariana aguda e AVC. Considerando ainda que essas mortes guardam uma correlaçáo com os respectivos casos diagnosticados totais existentes (i.e., por meio da letalidade entre os casos incidentes de doença) ${ }^{23,26,27}$, o tabagismo foi responsável por 470.666 casos diagnosticados de infarto agudo do miocárdio ou síndrome coronariana aguda, 59.509 casos diagnosticados de AVC, 378.594 casos de DPOC e 26.850 diagnósticos de câncer de pulmão. $\mathrm{O}$ custo total para tratar esses doentes, os quais obviamente incluem também os indivíduos que vieram a falecer, foi estimado em R \$ 24.776 milhóes, corrigido para o ano de 2013. E o custo direto médio de tratamento/assistência dessas doenças relacionadas ao uso do cigarro equivalente a uma morte foi de $\mathrm{R} \$ 0,264$ milhão (Tabela 1).

\section{Combinando Estudo I e Estudo II:}

Para cada centavo investido em marketing pela indústria do tabaco, o Brasil tem um gasto com tratamento de doenças relacionadas ao tabaco 1,927 vezes superior ao investido pela indústria (Tabela 1 e Figura 2). 
Tabela 1. Relação entre o custo direto médio do tratamento dos casos diagnosticados atribuíveis ao uso do cigarro e a parcela do lucro da indústria do tabaco revertida em ações de marketing

\begin{tabular}{|c|c|c|c|c|c|c|c|c|}
\hline \multirow[b]{2}{*}{ Doenças } & \multicolumn{3}{|c|}{$\begin{array}{l}\text { Estudo I "Parcela do lucro da indústria do } \\
\text { tabaco equivalente a } 1 \text { morte" * }\end{array}$} & \multicolumn{4}{|c|}{$\begin{array}{l}\text { Estudo II "Custo direto do tratamento dos casos } \\
\text { diagnosticados atribuídos ao uso do cigarro" * }\end{array}$} & \multirow{2}{*}{$\begin{array}{l}\text { Estudos I e } \\
\text { Il: Relação } \\
\text { "custo direto } \\
\text { médio do } \\
\text { tratamento" } \\
\text { vs "parcela do } \\
\text { lucro revertido } \\
\text { em ações de } \\
\text { marketing" }\end{array}$} \\
\hline & $\begin{array}{c}\text { Mortes } \\
\text { atribuíveis } \\
\text { ao consumo } \\
\text { de cigarro } \\
\text { legal }\end{array}$ & $\begin{array}{l}\text { Lucro total } \\
\text { (RS milhões) }\end{array}$ & $\begin{array}{c}\text { Parcela } \\
\text { do lucro } \\
\text { equivalente } \\
\text { a } 1 \text { morte } \\
\text { (RS milhões) }\end{array}$ & $\begin{array}{l}\text { Mortes } \\
\text { atribuíveis } \\
\text { ao cigarro }\end{array}$ & $\begin{array}{c}\text { Casos } \\
\text { diagnosticados } \\
\text { atribuíveis ao } \\
\text { cigarro }\end{array}$ & $\begin{array}{l}\text { Custo direto } \\
\text { tratamento } \\
\text { (RS milhões) \# }\end{array}$ & $\begin{array}{l}\text { Custo direto } \\
\text { médio de } \\
\text { tratamento } \\
\text { equivalente } \\
\text { a } 1 \text { morte } \\
\text { (RS milhões) \# }\end{array}$ & \\
\hline $\begin{array}{l}\text { Infarto agudo } \\
\text { do miocárdio/ } \\
\text { síndrome } \\
\text { coronariana aguda }\end{array}$ & 9.858 & 3.090 & na & 28.195 & 470.666 & 7.131 & na & na \\
\hline $\begin{array}{l}\text { Acidente } \\
\text { cerebrovascular }\end{array}$ & 6.780 & 3.090 & na & 10.812 & 59.509 & 1.872 & na & na \\
\hline $\begin{array}{l}\text { Doença pulmonar } \\
\text { obstrutiva crônica }\end{array}$ & 3.416 & 3.090 & na & 31.120 & 378.594 & 13.796 & na & na \\
\hline Câncer de pulmão & 2.479 & 3.090 & na & 23.762 & 26.850 & 1.976 & na & na \\
\hline Total & 22.533 & 3.090 & 0,137 & 93.889 & 93.5619 & 24.776 & 0,264 & 1,927 \\
\hline
\end{tabular}

Legendas: na=năo aplicável; * relacionados aos indivíduos com 35 anos ou mais de idade; \#ano de referência $2013^{33}$.

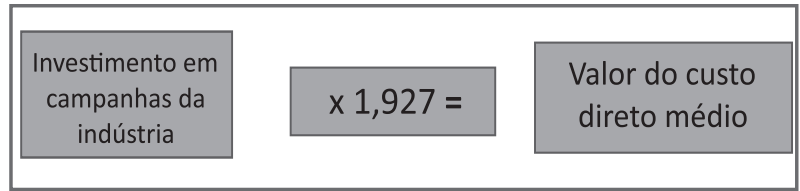

Figura 2. "Desproporção" entre o valor investido pela indústria do tabaco em ações de marketing e o que se gasta com tratamento

\section{DISCUSSÃO}

A CQCT/OMS estabelece referências para o controle do tabaco, a fim de reduzir de maneira contínua e substancial o seu uso e os seus impactos sanitário, social, ambiental e econômico. No entanto, suas medidas são continuamente minadas e obstruídas por estratégias da indústria do tabaco para manter sua capacidade de atrair jovens para o tabagismo e, assim, retroalimentar o seu mercado consumidor: seja por meio de utilização de brechas deixadas pela legislação nacional, seja por meio de violação das leis existentes, ou mesmo por açôes judiciais com intuito de reverter ou minimamente retardar a adoçáo de medidas efetivas para reduzir o consumo de seus produtos. Como exemplo recente dos esforços envolvidos com essa última estratégia, vale citar a norma da Anvisa que proibiu o uso de aditivos saborizantes em produtos de tabaco no ano de $2012^{34,35}$. A medida não chegou, contudo, a entrar em vigor em razão de uma liminar concedida pelo Supremo Tribunal Federal (STF) à Confederação Nacional da Indústria (CNI), que questionava se a Anvisa teria competência legal para determinar essa proibição.

O tabagismo é frequentemente aludido como uma doença pediátrica ${ }^{5}$. Não porque as consequências negativas para a saúde decorrentes do comportamento de fumar sejam restritas aos jovens, mas porque a iniciação ocorre na adolescência, época da vida marcada por intensas mudanças, pela busca de autoafirmação, rebeldia e transgressão, na qual o jovem busca experimentar novas atitudes e comportamentos ${ }^{9,14,20}$.

Embora a indústria do tabaco negue veementemente que dirija estratégias de marketing visando a atingir o público jovem, documentos internos provenientes de litígios internacionais revelaram que as empresas de tabaco o fizeram, e fazem, e que, de fato, dependem do mercado consumidor dos jovens para a sua sobrevivência em longo prazo ${ }^{36-39}$. Diversos estudos têm revelado de forma consistente que a exposição à publicidade/promoção do tabaco aumenta a probabilidade de os adolescentes começarem a fumar ${ }^{40-53}$, tendo sido, inclusive, constatada uma relação "dose-resposta" 52 .

Apesar de a legislação atual brasileira restringir a exposiçáo dos produtos derivados do tabaco à parte 
interna dos pontos de venda ${ }^{54}$, o ideal seria que a sua regulamentação nesses locais retirasse qualquer hipótese de visibilidade. Além disso, a experiência internacional/ nacional mostra que, sempre que publicidade, promoção e propaganda de produtos de tabaco sáo proibidas em determinados locais/meios de comunicação, há um investimento em marketing da indústria do tabaco para "burlar" essa restrição e/ou estimular a migração "ilegal" para mídias mais difíceis de serem controladas, tais como videogames, propaganda por celular e Internet ${ }^{55}$, o que sinaliza a importância do monitoramento permanente da percepçáo de todos os tipos possíveis de publicidade. A Internet se tornou, de fato, um campo de propagação do consumo de produtos de tabaco. É cada vez mais comum identificar a utilização de influenciadores digitais nas redes sociais fazendo propaganda de produtos legais $\mathrm{e}$ ilegais de tabaco ${ }^{56}$. Esse tema ganhou tanta sensibilidade que, na 8a Sessão da Conferência da Partes da CQCT/ OMS, realizada em 2018, os países membros decidiram estabelecer um grupo de trabalho para desenvolver diretrizes específicas para o desafio das açôes relacionadas ao Artigo 13 da Convenção, principalmente pelo seu impacto transfronteiriço catapultado pelos avanços tecnológicos das últimas décadas ${ }^{55}$.

Com a proibição das mídias tradicionais e a autorização da exposição dos produtos de tabaco apenas nos pontos de venda, houve também a "expansão física desses pontos de venda” em açóes publicitárias envolvendo grandes eventos musicais, tais como Rock in Rio e Lollapalooza ${ }^{57}$. Em particular, no caso da edição do Rock in Rio de 2017, foi instaurada Ação Civil Pública (ACP) por parte do Ministério Público do Estado do Rio de Janeiro (MPRJ) contra a empresa Souza Cruz, a Rock World (responsável pela realização do Rock in Rio) e a tabacaria Vega Fina pela identificação de um conjunto de atividades ilegais na promoçấo e propaganda de cigarros da marca Kent ${ }^{58}$. Entre as infraçốes constatadas, se destacavam: venda de kits compostos por maço e isqueiro elétrico com a logomarca do "Rock in Rio", utilização da venda ambulante no espaço do evento, a utilizaçáo de stand aberto luminoso e com a presença de marca do produto. $\mathrm{O}$ investimento realizado na implementação da tabacaria, segundo o MPRJ nos autos da ACP, demonstra a evidente ação de propaganda ilegal do produto. Foram investidos aproximadamente quatro milhóes de reais no evento, sendo que somente 45 mil reais foram destinados à aquisição do produto a ser vendido. Ao ter em mãos as informaçóes fornecidas pelos réus sobre o valor investido nessa campanha de marketing, o MPRJ poderia ter multiplicado esse valor por 1,927 para ter tido uma ideia do valor médio gasto correspondente para tratar as doenças associadas ao tabaco $(\mathrm{R}$ \$ 7.708.000,00). Esse seria um exemplo de aplicaçáo da metodologia descrita neste artigo para ajudar a estipular valores de ressarcimento.

\section{LIMITAÇÕES}

Encontrou-se uma relação constante de que o gasto do Brasil com tratamento/assistência de eventos associados ao tabaco é cerca de duas vezes maior do que o investimento em marketing da indústria do tabaco voltado a "capturar" novos fumantes. Essa relaçáo depende, obviamente, dos parâmetros que foram utilizados tanto no estudo I quanto no estudo II. Por exemplo, se o lucro da indústria do tabaco se modificar e/ou a proporção de fumantes de cigarros legais também não se mantiver constante ao longo do tempo, essa relação encontrada será alterada. Além disso, a própria equivalência atual encontrada entre o lucro revertido integralmente em despesa potencial com açóes de marketing e mortes de fumantes que contribuíram para a geração desse lucro total poderá não ser perfeitamente proporcional à equivalência esperada entre o valor efetivamente gasto em uma determinada campanha de marketing e mortes de fumantes, dependendo da fórmula utilizada para o cálculo das fraçóes da mortalidade por doenças específicas atribuíveis ao consumo de cigarros legais ${ }^{15,59}$. Essa relação constante também depende da evolução dos custos dos procedimentos no SUS em função, entre outros aspectos, da incorporação de novas tecnologias. No entanto, vale a pena ressaltar que a PNS, que ocorre a cada cinco anos, irá permitir a atualizaçấo periódica de grande parte dos parâmetros usados para o cálculo dessa relaçáo para o cenário brasileiro ${ }^{20}$. E o custo direto em assistência poderá ser corrigido pela inflação do ano para o qual se está realizando a análise, tendo, preferencialmente, o ano de realização da PNS como referência. Além disso, é importante notar que a atual baixa proporção de fumantes na população brasileira ${ }^{20}$, a relativa manutenção anual dos lucros relatados das empresas de tabaco $^{60}$ e a baixa taxa de incorporaçáo de novos procedimentos e/ou medicamentos para o tratamento das doenças selecionadas no artigo ${ }^{61}$ reforçam a hipótese de que a relação constante encontrada para 2013 não deve sofrer alteraçôes significativas com o passar dos anos.

\section{CONCLUSÃO}

No Brasil, continuam sendo identificadas ações de publicidade, propaganda e patrocínio por parte da indústria do tabaco em eventos musicais e por meio das redes sociais, o que é proibido. É fundamental que seja avaliada a ocorrência dessas ilegalidades e que seja mensurada a real dimensão do impacto negativo de açôes de marketing ilegais. Testar os limites do Estado de 
fiscalizar propaganda irregular faz parte das estratégias da indústria do tabaco para estimular, a qualquer custo, não somente a manutençáo do consumo de produtos de tabaco por tabagistas adultos, mas, principalmente, para incentivar a iniciação de jovens e crianças no tabagismo.

A metodologia descrita neste artigo pode servir de parâmetro para quantificar a responsabilização dos violadores da legislação nacional no que diz respeito a compensar parte dos custos associados ao tratamento de pacientes e aos programas de cessação ao fumo. Esse ressarcimento pode servir também como recurso para apoiar o desenvolvimento de pesquisas voltadas para quantificar o impacto positivo das campanhas sobre o sistema de saúde e a realização de açôes nacionais para desestímulo ao tabagismo.

\section{CONTRIBUIÇÕES}

André Salem Szklo, Felipe Lacerda Mendes, Tânia Maria Cavalcante e João Ricardo Viegas contribuíram substancialmente na concepção, na interpretação dos dados, na redação e na aprovação final da versão a ser publicada.

\section{DECLARAÇÃO DE CONFLITO DE INTERESSES}

Nada a declarar.

\section{FONTES DE FINANCIAMENTO}

Não há.

\section{REFERÊNCIAS}

1. Department of Health and Human Services (US). The health consequences of smoking: 50 years of progress. A report of the surgeon general. Atlanta (GA): Department of Health and Human Services, Centers for Disease Control and Prevention, National Center for Chronic Disease Prevention and Health Promotion, Office on Smoking and Health; 2014.

2. World Health Organization. WHO report on the global tobacco epidemic, 2019: Offer help to quit tobacco use [Internet]. Genebra: WHO; 2019 [cited 2020 Feb 6]. Available from: https://apps.who.int/iris/bitstream/han dle/10665/326043/9789241516204-eng.pdf?ua=1

3. Pinto M, Bardach A, Palacios A, et al. Burden of smoking in Brazil and potential benefit of increasing taxes on cigarettes for the economy and for reducing morbidity and mortality. Cad Saude Publica. 2019;35(8):e00129118. doi: http://dx.doi.org/10.1590/0102-311x00129118

4. Rezende LFM, Lee DH, Louzada MLDC, et al. Proportion of cancer cases and deaths attributable to lifestyle risk factors in Brazil. Cancer Epidemiol. 2019;59:148-57. doi: https://doi.org/10.1016/j. canep.2019.01.021

5. World Health Organization. International Statistical Classification of Diseases and Related Health Problems (ICD-10) [Internet]. 10th ed.; Version 2016. Geneva: WHO; 1996 [cited 2020 Feb 6]. Available from: https:// icd.who.int/browse10/2016/en\#/K27

6. World Health Organization. WHO Framework Convention on Tobacco Control [Internet]. Geneva: WHO; c2003 [cited 2020 Feb 6]. Available from: https:// www.who.int/fctc/text_download/en/

7. World Health Organization. History of the World Health Organization Framework Convention on Tobacco Control [Internet]. Geneva: WHO; c2009 [cited 2020 Feb 6]. Available from: https://apps.who.int/iris/bitstream/ handle/10665/44244/9789241563925_eng.pdf

8. World Health Organization. Neuroscience of psychoactive substance use and dependence [Internet]. Geneva: WHO; 2004. [cited $2020 \mathrm{Feb} 6$ ]. Available from: https://www.who. int/substance_abuse/publications/en/Neuroscience.pdf

9. Tobacco Company Marketing to kids [Internet]. Washington, DC: Campaign for Tobacco Free-Kids; 2020 Jan 13. [cited 2020 Feb 6]. Available from: https:// www.tobaccofreekids.org/assets/factsheets/0008.pdf

10. Ministério da Saúde (BR). Lei no 9.294, de 15 de julho de 1996. Dispóe sobre as restriçóes ao uso e à propaganda de produtos fumígenos, bebidas alcoólicas, medicamentos, terapias e defensivos agrícolas, nos termos do $\$ 4^{\circ}$ do art. 220 da Constituição Federal [Internet]. Diário Oficial da União, Brasília, DF; 1996 jul.16. Seção I, p. 13074 [acesso 2020 fev 6]. Disponível em: https://www.inca. gov.br/observatorio-da-politica-nacional-de-controle-dotabaco/legislacao-por-tema

11. Agência Nacional de Vigilância Sanitária. ResoluçãoRDC no 213, de 23 de janeiro de 2018. Dispóe sobre a exposiçẫo à venda e a comercialização de produtos fumígenos derivados do tabaco [Internet]. Diário Oficial da União, Brasília, DF; 2018 jan. 24 [acesso 2020 fev 6]. Disponível: em: https://www.inca.gov.br/observatorioda-politica-nacional-de-controle-do-tabaco/legislacaopor-tema

12. Szklo AS, de Souza MC, Szklo M, et al. Smokers in Brazil: who are they? Tob Control. 2016;25(5):564-70. doi: http://dx.doi.org/10.1136/tobaccocontrol-2015-052324

13. Levy D, de Almeida LM, Szklo A. The Brazil SimSmoke policy simulation model: the effect of strong tobacco control policies on smoking prevalence and smokingattributable deaths in a middle income nation. PLoS Med. 2012;9(11):e1001336. doi: https://doi. org/10.1371/journal.pmed.1001336

14. Instituto Brasileiro de Geografia e Estatística. Pesquisa nacional de saúde do escolar: 2015. Rio de Janeiro: IBGE; 2016. 
15. Szklo AS, Iglesias RM, de Souza MC, et al. Understanding the relationship between sales of legal cigarettes and deaths: a case-study in Brazil. Prev Med. 2017;94:55-9. doi: https://doi.org/10.1016/j.ypmed.2016.11.008

16. Goodchild M, Nargis N, Tursan d'Espaignet E. Global economic cost of smoking-attributable diseases. Tob Control. 2018;27(1):58-64. doi: http://dx.doi. org/10.1136/tobaccocontrol-2016-053305

17. Drope J, Schluger N, editors. The tobacco atlas [Internet]. 6th ed. Atlanta: American Cancer Society; 2018 [cited $2020 \mathrm{fev} 6$. Available from: https://tobaccoatlas.org/wpcontent/uploads/2018/03/TobaccoAtlas_6thEdition_ LoRes_Rev0318.pdf

18. Ipeadata [Internet]. Rio de Janeiro: Instituto de Pesquisa Econômica Aplicada; c2006. Sinopse macroeconômica; [acesso 2020 fev. 06]. Disponível em: http://www. ipeadata.gov.br/Default.aspx.

19. World Health Organization. Tobacco industry interference with tobacco control [Internet]. Geneva: WHO; c2008. [cited 2020 Feb 6]. Available from: https://www.who.int/tobacco/publications/industry/ interference/en/

20. Instituto Brasileiro de Geografia e Estatística. Pesquisa nacional de saúde 2013: percepçáo do estado de saúde, estilos de vida e doenças crônicas Brasil: grandes regióes e unidades da federação [Internet]. Rio de Janeiro: IBGE; 2015. [acesso 2020 fev 6]. Disponível em: https:// biblioteca.ibge.gov.br/visualizacao/livros/liv94074.pdf

21. Ministério da Economia (BR), Receita Federal [Internet]. Brasília, DF: Ministério da Economia; [data desconhecida]. Regimes e controles especiais; [acesso 2020 fev 6]. Disponível em: https://receita.economia.gov. br/orientacao/tributaria/regimes-e-controles-especiais.

22. Thun MJ, Carter BD, Feskanich D, et al. 50-Year trends in smoking-related mortality in the United States. N Engl J Med. 2013;368(4):351-64. doi: http://dx.doi. org/10.1056/NEJMsa1211127

23. DATASUS [Internet]. Brasília, DF: Ministério da Saúde (BR), Departamento de Informática do SUS; c2008. Informações de Saúde (TABNET); [acesso 2020 fev 6]. Disponível em: http://www2.datasus.gov.br/DATASUS/ index.php?area $=02$

24. Pichon-Riviere A, Augustovski F, Bardach A, et al. Development and validation of a microsimulation economic model to evaluate the disease burden associated with smoking and the cost-effectiveness of tobacco control interventions in Latin America. Value Health. 2011;14(5 Suppl 1):S51-9. doi: https://doi. org/10.1016/j.jval.2011.05.010

25. Centers for Disease Control and Prevention [Internet]. Atlanta: CDC; c2020. Smoking-attributable mortality, morbidity, and economic costs (SAMMEC). Smokingattributable expenditures (SAE); [update 2019 Feb 14; cited 2020 Feb 6]. Available from: https://chronicdata.
cdc.gov/Health-Consequences-and-Costs/SmokingAttributable-Mortality-Morbidity-and-Econo/ezab-8sq5

26. Bray F, Ren JS, Masuyer E, et al. Global estimates of cancer prevalence for 27 sites in the adult population in 2008. Int J Cancer. 2013;132(5):1133-45. doi: https:// doi.org/10.1002/ijc.27711

27. Azambuja MIR, Foppa M, Maranhão MFC, et al. Economic burden of severe cardiovascular diseases in Brazil: an estimate based on secondary data. Arq Bras Cardiol. 2008;91(3):148-55. doi: http://dx.doi. org/10.1590/S0066-782X2008001500005

28. SIGTAP: Sistema de gerenciamento da tabela de procedimentos, medicamentos e OPM do SUS [Internet]. Brasília, DF: Ministério da Saúde (BR), Departamento de Informática do SUS; [2007] [acesso 2020 fev 6]. Disponível em: http://sigtap.datasus.gov.br

29. BPS: Banco de Preços em Saúde [Internet]. Versão 3.1.33. Brasília, DF: Ministério da Saúde (BR). 1998 - [acesso 2020 fev 6]. Disponível em: https://bps.saude.gov.br/ login.jsf

30. Agência Nacional de Vigilância Sanitária [Internet]. Brasília, DF: [data desconhecida]. Câmara de Regulação do Mercado de Medicamentos; [acesso $2020 \mathrm{fev} 6$ ]. Disponível em: http://portal.anvisa.gov.br/cmed

31. Associação Médica Brasileira. Classificação Brasileira Hierarquizada de Procedimentos Médicos da Associação Médica Brasileira. São Paulo: AMB; 2017.

32. Pinto M, Ugá MAD. Custo do tratamento de pacientes com histórico de tabagismo em hospital especializado em câncer. Rev Saúde Públ. 2011;45(3):57582. doi: http://dx.doi.org/10.1590/S003489102011005000026

33. Instituto Brasileiro de Geografia e Estatística [Internet]. Rio de Janeiro: IBGE; [data desconhecida]. Índice Nacional de Preços ao Consumidor Amplo (IPCA); [acesso 2020 fev 6]. Disponível em: https://www.ibge. gov.br/estatisticas/economicas/precos-e-custos/9256indice-nacional-de-precos-ao-consumidor-amplo. html?t=downloads

34. Cenoz J. Proibição de aditivos em cigarros: 2 anos após julgamento do STF, medida segue protelada. 2020 jan 27 [acesso 2020 fev 6]. In: Blog ACT Promoção da Saúde [Internet]. Rio de Janeiro; São Paulo: ACT Promoção da Saúde. [data desconhecida]. Disponível em: http:// blog.actbr.org.br/controle-do-tabagismo/proibicao-deaditivos-em-cigarros-2-anos-apos-julgamento-do-stfmedida-segue-protelada/2257

35. Instituto Nacional de Câncer José Alencar Gomes da Silva. Aditivos em cigarros: notas técnicas para controle do tabagismo [Internet]. Rio de Janeiro: INCA; 2014. [acesso 2020 fev 6]. Disponível em: https://www.inca. gov.br/sites/ufu.sti.inca.local/files//media/document// aditivos-em-cigarros-notas-tecnicas-para-o-controle-dotabagismo.pdf 
36. Hastings G, MacFadyen L. A day in the life of an advertising man: review of internal documents from the UK tobacco industry's principal advertising agencies. BMJ. 2000;321(7257):366-71. doi: http://dx.doi. org/10.1136/bmj.321.7257.366

37. Cummings KM, Morley CP, Horan JK, et al. Marketing to America's youth: evidence from corporate documents. Tob Control. 2002;11:(Suppl 1):i5-i17. doi: http:// dx.doi.org/10.1136/tc.11.suppl_1.i5

38. Wen CP, Chen T, Tsai YY, et al. Are marketing campaigns in Taiwan by foreign tobacco companies targeting young smokers? Tob Control. 2005;14(Suppl 1):i38-44. doi: http://dx.doi.org/10.1136/tc.2004.007971

39. Truth Tobacco Industry Documents [Internet]. San Francisco: University of Carolina (UCSF). 2002 [cited 2020 Feb 06]. Available from: https://www. industrydocuments.ucsf.edu/tobacco/

40. Evans N, Farkas A, Gilpin EA, et al. Influence of tobacco marketing and exposure to smokers on adolescent susceptibility to smoking. J Natl Cancer Inst. 1995;87(20):1538-45. doi: https://doi.org/10.1093/ jnci/87.20.1538

41. Gilpin EA, Pierce JP, Rosbrook B. Are adolescents receptive to current sales promotion practices of the tobacco industry? Prev Med. 1997;26(1):14-21. doi: https://doi.org/10.1006/pmed.1996.9980

42. Feighery E, Borzekowski DL, Schooler C, et al. Seeing, wanting, owning: the relationship between receptivity to tobacco marketing and smoking susceptibility in young people. Tob Control. 1998;7(2):123-8. doi: http:// dx.doi.org/10.1136/tc.7.2.123

43. Lam T, Chung SF, Betson CL, et al. Tobacco advertisements: one of the strongest risk factors for smoking in Hong Kong students. Am J Prev Med. 1998;14(3):217-23. doi: https://doi.org/10.1016/ S0749-3797(97)00071-8

44. Mowery PD, Farrelly MC, Havilland ML, et al. Progression to established smoking among US youths. Am J Public Health. 2004;94(2):331-7. doi: http:// dx.doi.org/10.2105/AJPH.94.2.331

45. Slater SJ, Chaloupka FJ, Wakefield M, et al. The impact of retail cigarette marketing practices on youth smoking uptake. Arch Pediatr Adolesc Med. 2007;161(5):440-5. doi: http://dx.doi.org/10.1001/archpedi.161.5.440

46. Aitken PP, Eadie DR, Hastings GB, et al. Predisposing effects of cigarette advertising on children's intentions to smoke when older. Br J Addict. 1991;86(4):383-90. doi: http://dx.doi.org/10.1111/j.1360-0443.1991.tb03415.x

47. Pierce JP, Choi WS, Gilpin EA, et al. Tobacco industry promotion of cigarettes and adolescent smoking. JAMA. 1998;279(7):511-5. doi: http://dx.doi.org/10.1001/ jama.279.7.511

48. Biener L, Siegel M. Tobacco marketing and adolescent smoking: more support for a causal inference. Am J
Public Health. 2000;90(3):407-11. doi: http://dx.doi. org/10.2105/ajph.90.3.407

49. Gilpin EA, White MM, Messer K, et al. Receptivity to tobacco advertising and promotions among young adolescents as a predictor of established smoking in young adulthood. Am J Public Health. 2007;97(8):1489-95. doi: http://dx.doi.org/10.2105/AJPH.2005.070359

50. Pierce JP, Gilpin EA. A historical analysis of tobacco marketing and the uptake of smoking by youth in the United States: 1890-1977. Health Psychol. 1995;14(6):500-8. doi: http://dx.doi.org/10.1037//02786133.14.6.500

51. Lovato C, Linn G, Stead LF, et al. Impact of tobacco advertising and promotion on increasing adolescent smoking behaviours. Cochrane Database Syst Rev. 2003;(4):CD003439. doi:https://doi. org/10.1002/14651858.CD003439.pub2

52. DiFranza JR, Wellman RJ, Sargent JD, et al. Tobacco promotion and the initiation of tobacco use: assessing the evidence for causality. Pediatrics 2006;117(6):e1237-48. doi: https://doi.org/10.1542/peds.2005-1817

53. Pierce JP, Choi WS, Gilpin EA, et al. Validation of susceptibility as a predictor of which adolescents take up smoking in the United States. Health Psychol. 1996;15(5):355- 61. doi: https://doi.org/10.1037/02786133.15.5.355

54. Presidência da República (BR). Decreto 8.262, de 31 de maio de 2014. Altera o Decreto $n^{\circ} 2.018$, de $1^{\circ}$ de outubro de 1996, que regulamenta a Lei $\mathrm{n}^{\circ}$ 9.294, de 15 de julho de 1996 [Internet]. Diário Oficial da União, Brasília, DF; 2014 jun. 2. Seção I, p. 1. [acesso 2020 fev 6]. Disponível em: https://www.inca.gov.br/observatorioda-politica-nacional-de-controle-do-tabaco/legislacaopor-tema

55. WHO Framework Convention on Tobacco Control. Decision: FCTC/COP8: Tobacco advertising, promotion and sponsorship: depiction of tobacco in entertainment media [Internet]. In: Eighth session Conference of the Parties to the WHO Framework Convention on Tobacco Control; 2018 Oct 1-6; Geneva, Switzerland. [cited 2020 Feb 6]. Available from: https://www.who.int/fctc/cop/ sessions/cop8/FCTC_COP8(17).pdf?ua $=1$

56. Almeida LM, Silva RP, Santos ATC, et al. MC. Mists, vapors and other illusory volatilities of electronic cigarettes. Cad Saude Publica. 2017;33(Suppl 3):e00139615. doi: http://dx.doi.org/10.1590/0102-311X00139615

57. Lollapalooza Brasil [Internet]. Sáo Paulo: Lollapalooza, LLC; [data desconhecida] [acesso $2020 \mathrm{fev} 6$ 6]. Disponível em: https://www.lollapaloozabr.com/

58. Ministério Público do Estado do Rio de Janeiro (BR). Processo: 0204818-42.2019.8.19.0001. Açáo Civil Pública - Cláusulas abusivas/direito do consumidor. Autor: Ministério Público. Reú: Rock World S/A; Souza Cruz LTDA; Veja Fina Tabacaria Eireli. Decisão proferida 
em audiência especial realizada em setembro de 2019 na sala do Juízo da 6a Vara Empresarial da Capital. Fls. 383-385.

59. Rockhill B, Newman B, Weinberg C. Use and misuse of population attributable fractions. Am J Public Health. 1998;88(1):15-9. doi: https://doi.org/10.2105/ AJPH.88.1.15

60. British American Tobacco [Internet]. London: BAT; [date unknown]. Results and reporting; [cited 2020 Feb 06]. Available from: https://www.bat.com/latestresults

61. Comissão Nacional de Incorporação de Tecnologias em Saúde [Internet]. Brasília, DF: CONITEC; c2016-2020. Protocolos e diretrizes do Ministério da Saúde; 2019 set 24 [acesso 2020 fev 6]. Disponível em: http://conitec. gov.br/pcdt-em-elaboracao 